\title{
Biotech resilient in Q3
}

\author{
Stacy Lawrence
}

Despite the dismal economic climate, biotech stocks, financing, acquisitions and partnerships remained relatively solid. Although biotech indices have climbed only slightly since the beginning of 3Q07, they have

performed much better than the broader indices, which plunged. Large

\section{Biotech stock market performance}

Amidst economic and market turmoil, biotech indices solidly outperformed broader market indices.

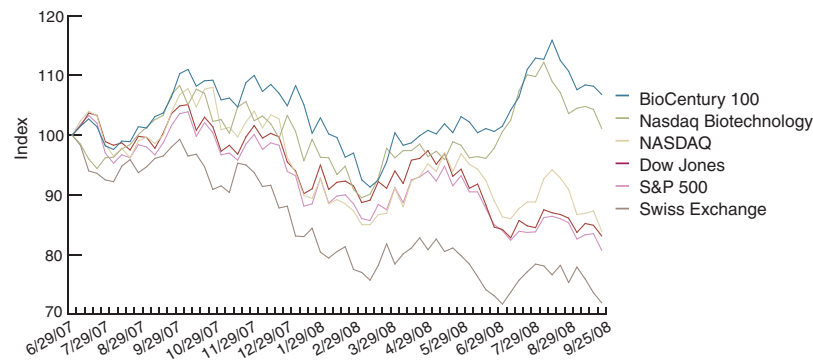

Source: $\mathrm{BCIQ}$ : BioCentury Online Intelligence, Multex

\section{Global biotech industry financing}

Total financing rebounded by $16 \%$ last quarter, driven by a $\$ 1.5$ billion private investment in public equities (PIPEs) for Australian biotech CSL.

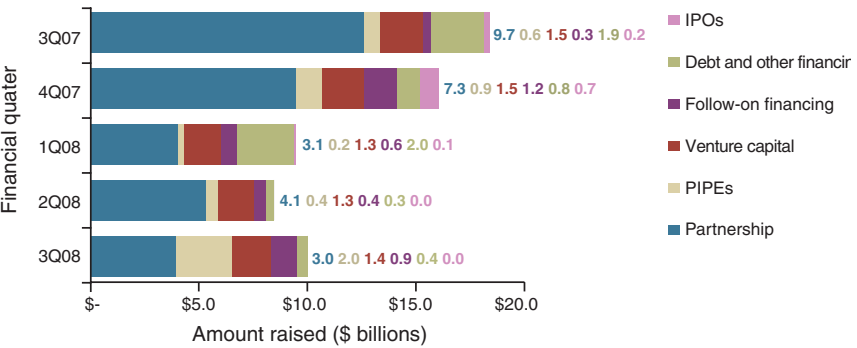

Partnership figures are for deals involving a US company. Source: $\mathrm{BCIQ}$ : BioCentury Online Intelligence, Burrill \& Company

Venture capital

\begin{tabular}{lclc} 
Company & $\begin{array}{l}\text { Amount invested } \\
\text { (\$ millions) }\end{array}$ & Round number & Date closed \\
\hline Portola & 130.0 & 3 & 9-Jul \\
\hline Pacific Biosciences & 100.0 & 5 & 14-Jul \\
\hline Proteolix & 79.0 & 3 & 8-Sep \\
\hline ESBATech & 62.5 & 2 & 7-Aug \\
\hline Anthera & 48.0 & 2 & 13-Aug \\
\hline Intercept & 45.5 & 1 & 29-Jul \\
\hline Proteostasis & 45.0 & 1 & 25-Aug \\
\hline
\end{tabular}

Licensing/collaboration

\begin{tabular}{llc} 
Researcher & Investor & Value $\mathbf{( \$ \text { millions) }}$ \\
\hline Actelion & GlaxoSmithKline & $3,200.0$ \\
\hline Cellzome & GlaxoSmithKline & $1,500.0$ \\
\hline Medivation & Pfizer & 725.0 \\
\hline PDL BioPharma & Bristol-Myers Squibb & 710.0 \\
\hline Immunomedics & Nycomed & 620.0 \\
\hline Ablynx & Merck Serono & 490.6 \\
\hline PTC Therapeutics & Genzyme & 437.0 \\
\hline Amgen & Ortho-McNeil-Janssen & 435.0 \\
\hline CV Therapeutics & Menarini & 385.0
\end{tabular}

Deal description Co-develop and co-commercialize phase 3 insomnia candidate almorexant outside of Japan Discover, develop and market kinase-targeted therapeutics to treat inflammatory diseases Develop and commercialize phase 3 dimebon to treat Alzheimer's disease Co-develop and commercialize phase 1 elotuzumab (HuLuc63) to treat multiple myeloma Develop and commercialize phase $1 / 2$ subcutaneous veltuzumab in non-cancer indications Co-discover and co-develop nanobodies against two undisclosed targets in cancer and immunology Develop and commercialize phase 2b PTC124 to treat genetic diseases due to nonsense mutations Develop and commercialize a phase 1 undisclosed human antibody against nerve growth factor (NGF) Develop and commercialize approved Ranexa ranolazine in select areas outside the US to treat stable angina pectoris

\begin{tabular}{llcc}
$\begin{array}{l}\text { Mergers and acquisitions } \\
\text { Target }\end{array}$ & Acquirer & Value $\mathbf{\$}$ billions) & Date announced \\
\hline Genentech & Roche & 43.7 & 21-Jul \\
\hline Talecris Biotherapeutics & CSL & 3.1 & 12-Aug \\
\hline Sciele Pharma & Shionogi & 1.1 & $2-S e p$ \\
\hline Speedel & Novartis & 0.9 & $10-J u l$ \\
\hline Lev Pharmaceuticals & ViroPharma & 0.6 & 15-Jul \\
\hline
\end{tabular}

bids to acquire Genentech (S. San Francisco, CA, USA) and ImClone (New York) also generated interest in the sector. Financing is unlikely to outstrip the $\$ 53$ billion high in 2007 , but last quarter actually managed

\section{Global biotech venture capital investment}

VC financings actually rose the last two quarters from a low in $1 \mathrm{Q} 08$.

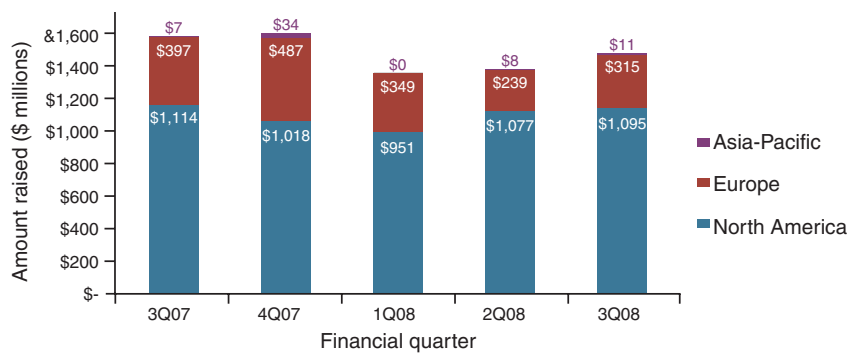

Source: $\mathrm{BCIQ}$ : BioCentury Online Intelligence

\section{Global biotech initial public offerings}

Not a single biotech initial public offering (IPO) made it out last quarter.

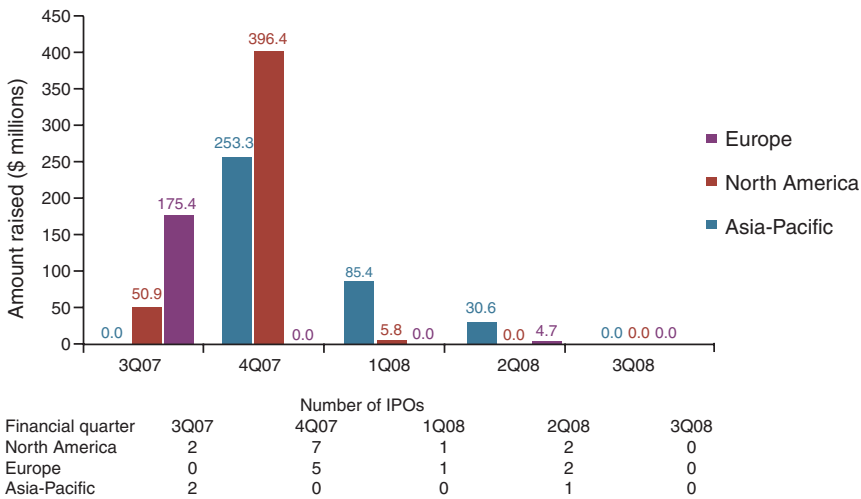

Source: $\mathrm{BCIQ}$ : BioCentury Online Intelligence

Source: $\mathrm{BCIQ}$ : BioCentury Online Intelligence, Deloitte Recap

Stacy Lawrence is Senior Writer, BioCentury 